\title{
A novel approach to analyze total amino acids contents of food samples by computational image scanning densitometry
}

\author{
W. Uz-Zaman, R. Rehman*, J. Zafar \\ Institute of Chemistry, University of the Punjab, Lahore-54590, Pakistan
}

Received November 17, 2015; Accepted February 27, 2019

In the current work, computational image scanning densitometry technique was used instead of conventional and traditional instruments like IC, HPLC, GC and UV/visible spectroscopy for quantification of micro samples of amino acids (lysine hydrochloride, $\beta$-alanine, asparagine monohydrate, glutamic acid). The general reaction between locating reagent and amino acid is:

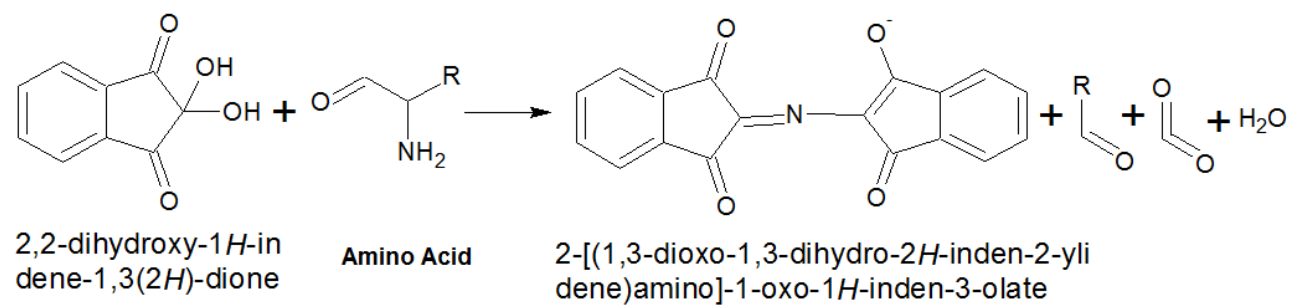

This technique has accuracy at the parts per billion level and precision as indicated from percent recovery from sample analysis, which is quite good. Due to certain limits associated with conventional techniques, this technique may be presented as a progressive and important event in the history of evaluation at micro level.

Keywords: amino acids, densitometry, micro level.

\section{INTRODUCTION}

Absorption spectroscopy is a sort of estimation of an interaction by the relation between electromagnetic radiation and content of atoms of a chemical substance. Pharmaceutical analysis usually involves atomic absorption, ultraviolet/visible and infrared spectroscopy. Colorimetry is basically a spectrophotometric measurement in the visible region which is apt to be called "colorimetry" only when taking into consideration human perception of color. There are several techniques which employed and developed AAS and ICP, but in developing countries most of the analytical labs cannot afford such costly techniques. As a result, the only novel technique which is left is spectrophotometry which is used around the world for estimation at micro level. Despite the fact, there are several benefits along with few disadvantages. For instance, the expected results in respect of accuracy and precision are not achieved in the case of micro samples. Likewise, spectrophotometric technique cannot be laid off directly [1], as is the matter of coagulation just like $\mathrm{Ni}^{2+}$ with DMG. Similarly, atomic absorption spectrometry (AAS) is a well accepted and useful technique for the estimation of metals in minute

\footnotetext{
* To whom all correspondence should be sent:

E-mail: grinorganic@yahoo.com
}

amounts, but still the method has to require many developmental series concerning getting more in accuracy and ease of operation and, predominantly, it requires a lot of improvement for estimation up to a certain limit [2]. In the laboratory practice of protein purification a comparatively quick and discriminating procedure to quantify (estimation of LOD) the polymers of amino acids is frequently needed, this protocol eliminates the requirement of this kind of estimation [3]. To overcome the limitation of spectrophotometry, researchers around the world are keenly working and trying to elaborate particular methods for the accurate and precise analysis of micro quantities of samples [4]. Teasdale et al. advanced this approach for sulfur containing compound evaluation [5].

A quality-level scanner and imaging software was utilized to make the densitometric calculations. Abraham et al. used this protocol for the separation of allantoin on the thin layer chromatogram and dimethylaminobenzaldehyde was sprinkled on the spot [6]. Densitometer was used to read the yellow colored spot. As the matter of bottom value for quantification was $0.1 \mathrm{mg}(0.5 \mathrm{mg}=100 \mathrm{ml})$ for every spot. Quantization of allantoin in urine, serum and lymph can be done through this method which is an advantage. The practice of scanning densitometry for the examination of dyes in food products was probed by Ueno et al. [7]. 
W. Uz-Zaman et al.: A novel approach to analyze total amino acids contents of food samples by computational image... Pharmacologically active curcuminoids were isolated by Paramasivam et al. from the rhizome of curcuma longa [8]. By using extraction (Soxhlet extraction) the curcuminoids were separated from the rhizomes of turmeric. Crystallization and column chromatography were used for the separation and spectroscopic analysis for identification. TLC aluminum plates with silica gel spread on the plate were used in this method. Curcuminoids were analyzed using the densitometric method in assimilation-deliberation mode at $425 \mathrm{~nm}$. To examine the percentage of these three curcuminoids, 7 different kinds of germplasm of turmeric were detected. Mohammad and Zehra have developed a chromatographic system containing a mobile phase of boratephosphate buffer with $\mathrm{pH} 2.3$ and a silica layer saturated with an anionic surfactant as a stationary phase [9]. It was shown as the most apt system for the mutual separation of $d l$-tryptophan and $l$ histidine. Stability, lowest detectable amount, reproducibility and retardation factor values of these two amino acids were estimated. Silica TLC plates and silica gel 60F254HPTLC plates were used for the evaluation and comparison of chromatographic parameters including separation factor and dissolution factor for the separation of $l$ histidine and $d l$-tryptophan.

Amino acids are carbon compounds containing both nitrogenous compounds and carboxylic groups. Apart from glycine, every other amino acid has chirality and is optically active [10]. In the present years it is found that amino acids not only exist as single molecules inside the cell but they are also considered as a circulator of the protein phosphorylation cascade and of gene expression. Moreover, amino acids act as forerunners for preparation of steroidal compounds and least atomic mass nitrogen conatining compounds, both have huge biological significance. The bodyrequired level of amino acids and their related end products (e.g., nitric oxide, polyamines, uric acid, glutathione, steroidal chemical messenger) are needed for many functions in the body. Inflated concentrations of amino acids and their resulting compounds (e.g., $\mathrm{NH}_{3}$, asymmetric dimethyl arginine and homocysteine) are fatal and responsible for abnormalities related to neurons, higher potential of oxidation, and diseases associated to heart. Hence, an optimal, apt and suitable balance among amino acids in the diet taken is critical and crucial for the body homeostasis [11]. Relative examination of amino acids at $\alpha$-position is done by treating with ninhydrin (2,2-dihydroxy-1,3,-indandione). The product is dehydrogenated alcohol which contains an atom of carbon less than the amino acid having $\alpha$-position along with $\mathrm{CO}_{2}$ in stoichiometric ratio, also formed different values of hydrindantin, $\mathrm{NH}_{3}$ and a color imparting compound called Ruhemann's Purple (diketohydrindylidenediketohydrindamine). Ruhemann's Purple pigment tends to serve as a basis of quantitative analysis along with estimation of $\alpha$-amino acids [12]. In amino acid sample analysis, total amino acid contents were determined by various methods like chromatography, HPLC, spectroscopy, etc., instead of reporting individual amino acid contents. This is the main limitation in their analysis.

In the current work, instead of the use of conventional and traditional instrumentation like IC, HPLC, GC and UV/visible spectroscopy, a technique was used for quantification of micro samples of amino acids (lysine hydrochloride, $\beta$ alanine, asparagine monohydrate, glutamic acid). Particular media (silica plate and TLC) were used to shift the measured volume $(0.5 \mu \mathrm{L})$ of sample and standard. The medium may contain a reagent (Ninhydrin reagent) impregnated, succeeding deposit the reagent, after enough drying, digital scan of developed spot is transferred inside the computer by using a high-quality scanner at 300 dpi, RGB mode of 24 bit. By using appropriatlely and specially developed software the image is quantified by making the spot well inside the selection marquee. The GBR, (green, blue, red) component of every visual display (a mixed area of illumination) from the periphery and added amounts up to ultimate color innermost quantity are measured by the software illustrated below. A model for subtractive color presentation is the RGB color space, each of RGB components is at its maximum value shown by pure white color (eight bit per component $1 / 4255$ ), similarly $R^{1 / 4} \mathrm{G}^{1 / 4} \mathrm{~B}^{1} / 4128$ indicated grey color, $\mathrm{R}^{1 / 4} \mathrm{~B}^{1 / 4} / \mathrm{G}^{1} / 40$ showed pure black color. The issue we are concerned with is density measurement; it only required a simple difference from the highest value (two hundred and twenty five) that will provide you with one fourth of RGB for White, one twenty eighth for Grey, and one two hundred and fifty fifth for Black. This technique is fundamentally applied for some other colors. A dusky color or, in other words, a higher intensification of component under examination, will cause a high intensity of color. Moreover, there are more than thousand visual units within the image of a mark (a $1 \mathrm{~mL}$ blot on TLC encloses a circle of point two" diameter, which forms a three dpi image enclosing almost twenty eight hundred visual units), the ultimate accruing color intensity of the complete blot consequence into six numerals [13]. 
W. Uz-Zaman et al.: A novel approach to analyze total amino acids contents of food samples by computational image...

Concentration of analyte in the sample is calculated by plotting the calibration curve. It is noteworthy that the selectivity depends not on the instrumental methodology but on the reagent; therefore be careful when selecting the developing reagents. Extremely small sample volume is used; colored and black precipitation can be quantified in contrary to spectrophotometry; for measurement no costly instrument is required; by using a laptop and a digital camera this technique can be portably used; all these are few advantages of this technique.

\section{EXPERIMENTAL}

\section{Apparatus and instrumentation}

For development of the spots, a $\mu \mathrm{L}$ pipette and TLC foil (Merck, TLC aluminum membrane, $(20 \times$ $20 \mathrm{~cm}$, silicon dioxide gel 60f254) was utilized. For transcribing into the digital form of the image of the mode, HP thirty six hundred and seventy CCD contemplative smooth level color scanner was used.

\section{Quantification of amino acids}

Amounts of $0.938 \mathrm{~g}, 1.877 \mathrm{~g}, 2.283 \mathrm{~g}$ and 1.114 $\mathrm{g}$ of glycine, asparagine monohydrate, lysine monohydrochloride and $\beta$-alanine, respectively, were taken to make $25 \mathrm{~mL}$ of $0.5 \mathrm{M}$ solution of each one. Ninhydrin reagent was prepared by mixing 1.0 $\mathrm{g}$ of the reacting compound in $10 \mathrm{~mL}$ of pure ethanol. By making different concentrations of the standard solution mentioned, standards $(0.5 \mathrm{M}-$ $0.1 \mathrm{M})$ were prepared.

\section{Spot development}

By using the micro pipette $0.5 \mu \mathrm{L}$ of ninhydrin reagent followed by each standard was applied on TLC plates $(35 \mathrm{~cm})$ at suitable distances. The plates were placed inside an oven at $80^{\circ} \mathrm{C}$ for $10 \mathrm{~min}$ to develop the brownish red spot after short drying, as shown in Fig. 1. The general reaction is shown in Fig. 2.

To measure the color density of each spot, the TLC sheet was scanned on a high-resolving scanner and the consequent image was estimated in software. Between the concentration and color consistency, the gauging line was dragged, and then to measure the intensity of the amino acids inside the sample, the calibration data were used. Moreover, for comparison of optical densitometric technique with visible spectrophotometry, $2 \mathrm{~mL}$ from each solution in the range from $0.1-0.5 \mathrm{M}$ of each amino acid were taken in separate test tubes and $3 \mathrm{~mL}$ of ninhydrin reagent were added. These solutions were heated in an oven at $80^{\circ} \mathrm{C}$ for $10 \mathrm{~min}$ and the absorbance was read at $624 \mathrm{~nm}$.

While mentioning the application of densitometry using ninhydrin as a reagent for detection and estimation of amino acids, samples of wheat flour, jatropha seed cake (seed cake left after the extraction of oil from it) and grinded rice were taken, their sample preparation involves the lysis and extraction of proteins by treatment with $6 \mathrm{M}$ $\mathrm{HCl}$ followed by incubation in a microwave for about $10 \mathrm{~min}$. Afterwards the sample was neutralized (to $\mathrm{pH}=8.03$ ) with liquid ammonia.

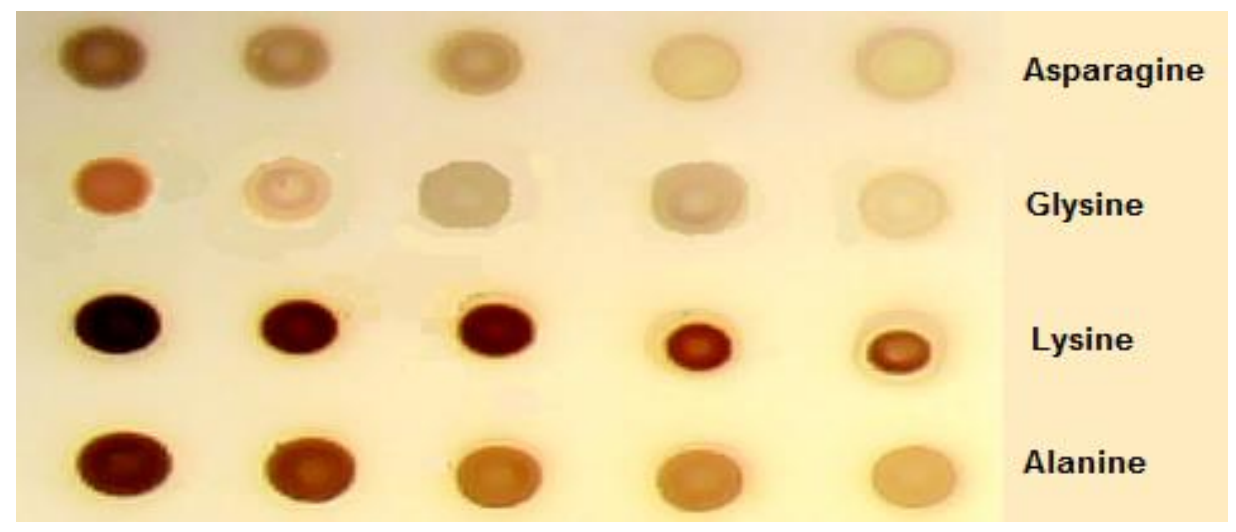

Fig. 1. Developed spots of amino acids with ninhydrin for densitometric analysis. 


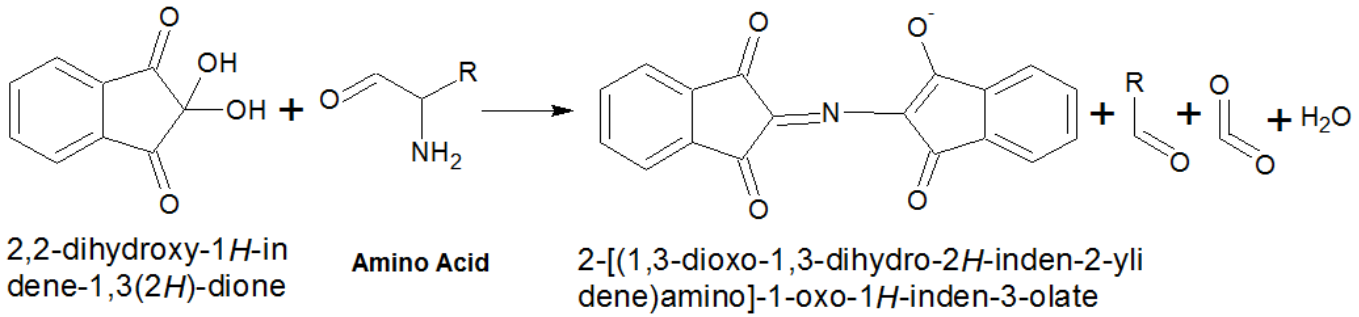

Fig. 2. Schematic reaction of amino acid with reagent.

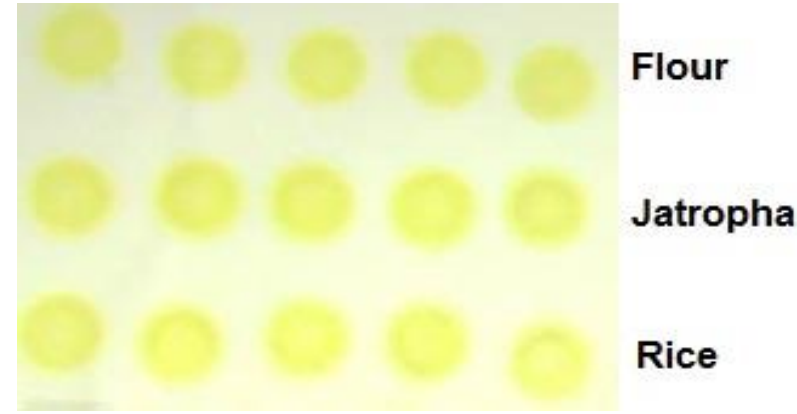

Fig. 3. Developed replicate spots of amino acids contents of samples.

Each sample was diluted by taking $1 \mathrm{~mL}, 2 \mathrm{~mL}$, $3 \mathrm{~mL}, 4 \mathrm{~mL}$ and $5 \mathrm{~mL}$ from the stock solution and made up to a total volume of $5 \mathrm{~mL}$ with distilled water. Each sample was then placed on a TLC plate. After adding ninhydrin reagent and by following the same procedure as marked out before, the brownish red color spots were developed and then quantified with the algorithm designed for it.

\section{RESULTS AND DISCUSSION}

The estimation of total amino acid can be carried out by various methods - the classical method and the spectrophotometric method. Due to their low precision at micro level, difficult handling and cost these methods are not appropriate. Quantification is a very sensitive issue at micro level in food samples. All over the earth efforts are being made to find out a reasonable inexpensive and authentic methodology for the estimation of metal content at micro level in the sample.

In this study, an approach was advanced at micro level for absolute consideration of amino acids similar to $\beta$-alanine, lysine monohydrochloride, asparagine monohydrate and glutamic acid. The fascination of this protocol is its being easy to handle and not needing formal apparatus. It can be used with great precision at very low levels for the quantification of toxic metals. Other advantages include: use of minor amount; quantification of the color coagulation; intensified delicacy; perfection.

The gauging equalization for $\beta$-alanine, lysine monohydrochloride, asparagine monohydrate and glutamic acid reveal an impressive correlation coefficient $\left(\mathrm{R}^{2}>0.9\right)$. The calibration data for determination of amino acids are shown in Table 1. It indicates that calibration line can be made with any of the selected amino acids giving similar results with total amino acid contents of samples being analyzed, as shown in Table 2 . For checking the applicability of this method, total amino acid contents of wheat flour, jatropha and rice were determined using calibration line of $\beta$-alanine and spiking its standard solution in these samples for estimating percent recovery as shown in Fig.3. This method was also compared to standard visible spectrophotometry using standard solutions ranging from 0.1 to $0.5 \mathrm{M}$ of $\beta$-alanine. From each solution $2 \mathrm{~mL}$ were taken in separate test tubes and $3 \mathrm{~mL}$ of ninhydrin reagent were added. The solutions were heated in the oven at $80^{\circ} \mathrm{C}$ for $10 \mathrm{~min}$ and then the absorbance was read at $624 \mathrm{~nm}$. Same was done with wheat flour, jatropha and rice samples for analysis comparison.

Table 2 indicates that more than $98.5 \%$ have been recovered. So it is safely concluded that this method can be applied on industrial scale for continuous monitoring of staples samples for their total amino acid contents. The results indicated that the computational image scanning densitometry method is comparable in accuracy with the standard method. 
W. Uz-Zaman et al.: A novel approach to analyze total amino acids contents of food samples by computational image...

Table 1. Calibration data for determination of amino acids.

\begin{tabular}{|c|c|c|c|c|}
\hline \multirow{2}{*}{$\begin{array}{c}\text { Concentration } \\
(\mathrm{ng} / \mathrm{mL})\end{array}$} & \multicolumn{3}{|c|}{ Color density } \\
\cline { 2 - 5 } & $\beta$-Alanine & $\begin{array}{c}\text { Lysine } \\
\text { monohydrochloride }\end{array}$ & $\begin{array}{c}\text { Asparagine } \\
\text { monohydrate }\end{array}$ & Glutamic acid \\
\hline 1 & 31418 & 61418 & 44180 & 69418 \\
\hline 2 & 94517 & 124517 & 67517 & 142517 \\
\hline 3 & 142107 & 162107 & 115107 & 238110 \\
\hline 4 & 190110 & 200110 & 163110 & 256985 \\
\hline 5 & 218985 & 238985 & 191985 & 297193 \\
\hline 6 & 259193 & 279193 & 232193 & $\mathrm{y}=43722 \mathrm{x}+46027$ \\
\hline $\begin{array}{c}\text { Calibration } \\
\text { equation }\end{array}$ & $\mathrm{y}=44579 \mathrm{x}+26.8$ & $\mathrm{y}=42008 \mathrm{x}+30693$ & $\mathrm{y}=38899 \mathrm{x}-465.2$ & 0.967 \\
\hline $\begin{array}{c}\text { Correlation } \\
\text { coefficient }\left(\mathrm{R}^{2}\right)\end{array}$ & 0.986 & 0.9913 & 0.9925 & \\
\hline
\end{tabular}

Table 2. Concentration of total amino acids in food samples.

\begin{tabular}{|c|c|c|c|c|c|c|}
\hline \multirow{2}{*}{ Sample No } & \multirow{2}{*}{ Sample name } & \multicolumn{2}{|c|}{$\begin{array}{c}\text { Average concentration } \\
\mathrm{mg} / 100 \mathrm{~g}\end{array}$} & $\begin{array}{c}\text { Standard } \\
\text { deviation } \pm\end{array}$ & $\begin{array}{c}\text { Percent } \\
\text { recovery }\end{array}$ & $\begin{array}{c}\text { Percent } \\
\text { error }\end{array}$ \\
\cline { 3 - 6 } & & $\begin{array}{c}\text { Found by } \\
\text { spectroscopy }\end{array}$ & $\begin{array}{c}\text { Found by } \\
\text { densitometry }\end{array}$ & & & \\
\hline 1. & Wheat flour & 71.231 & 71.536 & 0.005508 & 99.2 & 0.7 \\
\hline 2. & Jatropha & 77.962 & 78.723 & 0.000577 & 99.3 & 0.9 \\
\hline 3. & Rice & 77.581 & 77.357 & 0.00026 & 98.6 & 1.3 \\
\hline
\end{tabular}

\section{CONCLUSION}

This work has exhibited that blot test utilizing scanning is more appropriate for computational quantification in many aspects like time, handling and cost as compared to the conventional techniques. This technique has parts per billion level accuracy and precision as indicated by percent recovery from sample analysis, which is quite good. Due to certain limits associated with conventional techniques, this technique may be presented as a progressive and important event in the history of evaluation at micro level.

\section{REFERENCES}

1. P. Nagaraja, H. S. Yathirajan, H. R. Arunkumar, R. A. Vasantha, J. Pharmaceut. Biomed., 29(1-2), 277 (2002).
2. S. Olsen, L. C. R. Pessenda, J. Ruizicka, E. H. Hansen, Analyst, 108, 1289 (1983).

3. M. Bradford, Anal. Biochem., 72, 248 (1976).

4. S. Kundu, M. Mundal, A. Pal, Talanta, 58(5), 935 (2002).

5. P.R. Teasdale, S. Hayward, W. Davison, Anal. Chem., 71(11), 2186 (1999).

6. J. Abraham, F. A. Simeone, R. W. Hopkins, Anal. Biochem., 70(2), 377 (1976).

7. E. Ueno, T. Ohno, H. Oshima, Shokuhin Eiseigaku Zasshi, 39(4), 286 (1998).

8. M. Paramasivam, R. Poi, H. Banerjee, A. Bandyopadhyay, Food Chem., 113(2), 640 (2009).

9. A. Mohammad, A. Zehra, Colloid. Surfaces A, 301(1-3), 404 (2007).

10. F. Galli, Amino Acids, 32, 497 (2007).

11. G. Wu, Amino Acids, 37, 1 (2009).

12. C. B. Bottom, S.S. Hanna, D. J. Siehr, Biol. Chem., 176, 367 (1948).

13. J. Anwar, W. Uz-Zaman, U. Shafique, M. Salman, Anal. Lett, 43(2), 367 (2010). 